EPJ Web of Conferences 47, 15002 (2013)

DOI: $10.1051 /$ epjconf/20134715002

(C) Owned by the authors, published by EDP Sciences, 2013

\title{
A new low proper motion catalogue of bright M-dwarfs
}

\author{
James Frith ${ }^{\mathrm{a}}$ and David Pinfield
}

University of Hertfordshire

\begin{abstract}
We present a new catalogue of 1336 bright $(\mathrm{K}<9)$, low proper motion, $\mathrm{M}$ dwarfs from the PPMXL catalogue. These M dwarfs cover the entire sky but the galactic plane was excluded to avoid crowding and reddened stars. Spectra taken of a random sample of the targets and spectral estimations made using colours of the sample show a spectral range K8-M5.
\end{abstract}

\section{INTRODUCTION}

Low mass stars have become of interest in recent years for their applications to exoplanet research especially because of their unique properties of having short orbital periods for planets lying in the habitable zone as well as favourable contrast ratios between the planet and the host star. Though $\mathrm{M}$ dwarfs are the most numerous type of stars in the Galaxy, because of their colour and low luminosity they often can be confused for reddened stars or distant red giants. Because of this their identification in large surveys has been difficult. With exoplanet research now moving from the time of discovery to the time of characterization, methods to obtain information about the exoplanet's atmosphere can benefit from bright $\mathrm{M}$ dwarf stars with transiting exoplanets and currently only a handful exist. The first step towards having a comprehensive list of transiting exoplanets around $\mathbf{M}$ dwarfs for characterization is to identify all potential bright $\mathrm{M}$ dwarf host stars in the sky and, as of yet, this catalogue doesn't exist. Other efforts towards an all sky, bright $\mathrm{M}$ dwarf catalogue have been made recently by [1]. This sample was created from the LSPM catalogue and supplemented with Tycho-2 objects but is limited at the low proper motion range due to the SUPERBLINK lower proper motion limit of 40 milli arcseconds. In an effort to explore this lower proper motion regime and expand on this existing sample, we have chosen to use the Position and Proper Motion Extended-L (PPMXL) [2] catalogue which is a combination of the 2MASS [3] and USNO-B1 [4] catalogues. PPMXL provides the 2MASS and USNO-B1 objects with absolute proper motions, using the Internation Celestial Reference System (ICRS) [5]. The 2MASS JHK band photometry is also combined with the USNO-B1 BVRI bands so the catalogue provides a wealth of photometric and astrometric data useful to define the M Dwarf sample. We've optimized a selection approach that combines reduced proper motion as an analogue to absolute magnitude and colour cuts using the many photometry bands available in PPMXL.

\section{GUIDING SAMPLES}

Several M dwarf samples exist in the literature that have been observed spectroscopicly and photometrically across many bands so some of these samples were used as guides when defining the M dwarf selection. Three M dwarf samples were used. One sample was produced by [6] in a spectroscopic survey for cool stars within 100 parsecs of the sun. Another is the Palomar/MSU spectroscopic

\footnotetext{
${ }^{a}$ e-mail: j.frith@herts.ac.uk
}

This is an Open Access article distributed under the terms of the Creative Commons Attribution License 2.0, which permits unrestricted use, distribution, and reproduction in any medium, provided the original work is properly cited. 


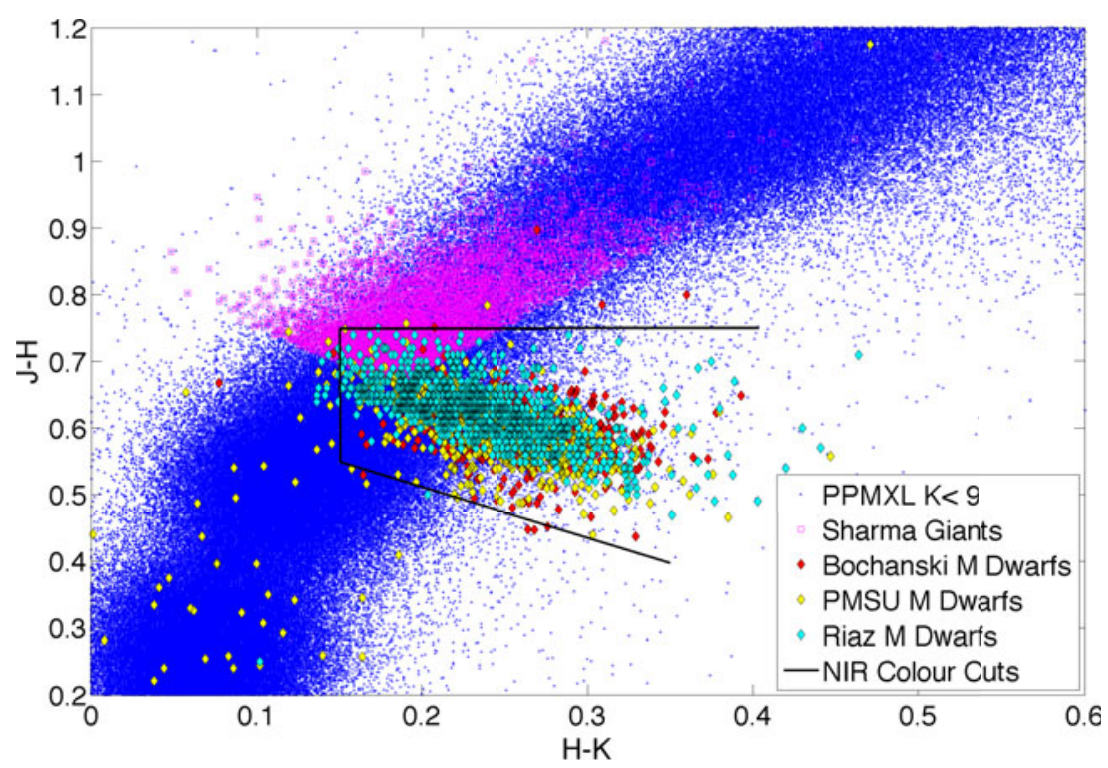

Figure 1. Colour colour plot showing the defined $M$ giants used for the reduced proper motion cuts where no known giants were available.

survey [7] which is based on spectra obtained to find M dwarfs in the Third Catalogue of Nearby Stars (CNS3) catalogue [8] which contained objects within 25 parsecs of the Sun. The third M dwarf selection was done by [9] and was compiled to find active M dwarfs using X-ray observations from ROSAT. All 3 catalogues were cross-matched with PPMXL with a cone radius of 2 arcseconds and the matched objects used as comparisons to our sample.

Distant $\mathrm{M}$ giants were a major source of potential contamination so a guiding sample of giants was also used from Famaey et al [10]. This sample consists of giants selected using known distances and absolute magnitudes from the Hipparcos catalogue [11]. $\mathrm{K}$ and $\mathrm{M}$ giants were selected using the absolute magnitude and V-I colour cuts. Though the majority of the sample consists of K giants, there are a significant number of $\mathrm{M}$ giants identified that were used below when attempting to remove potential giant contamination. These objects were also cross matched with PPMXL so all guiding samples had proper motion estimates mapped onto the ICRS. Not all photometric bands were available in the giant sample defined in the Hipparcos giants so colour cuts designed to select the M giant population were applied to the PPMXL targets and an internally defined giant sample was created. Our giant sample was defined using cuts from Sharma et al [12].

These cuts were applied to areas of sky avoiding the galactic plane to reduce reddened stars. As seen in Fig. 1, these cuts slightly overlap the region of $\mathrm{M}$ dwarfs in the colour-colour plot but the majority of the sample is well separated from the M dwarf colour space. This overlap illustrates how colour cuts alone don't completely isolate an $\mathrm{M}$ dwarf or $\mathrm{M}$ giant population and how more cuts are necessary to remove the giants for our selection.

The resulting samples were used as guides for the reduced proper motion selection below.

\subsection{Colour selections}

The initial colour selection was done using the 2MASS near-infrared JHK bands. These cuts were made to select the $\mathrm{M}$ dwarfs from the $\mathrm{M}$ giants as well as limit $\mathrm{K}$ dwarf contamination. The guiding samples were used to define the colour regions where most $\mathrm{M}$ dwarfs lie as can be seen in Fig. 1. The resulting 


\section{Hot Planets and Cool Stars}

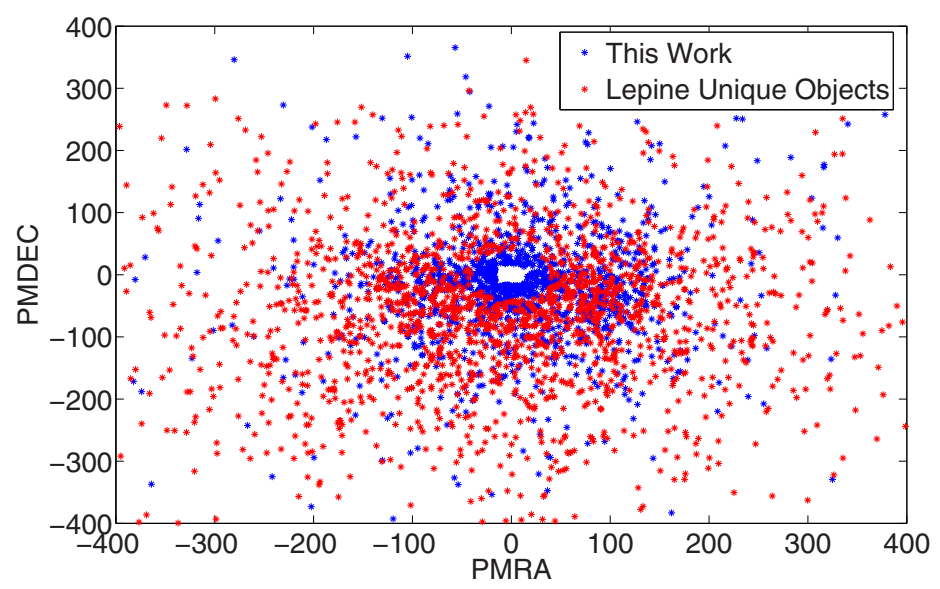

Figure 2. Proper Motion Plot showing the unique objects from each catalogue and the low proper motion region this work identifies.

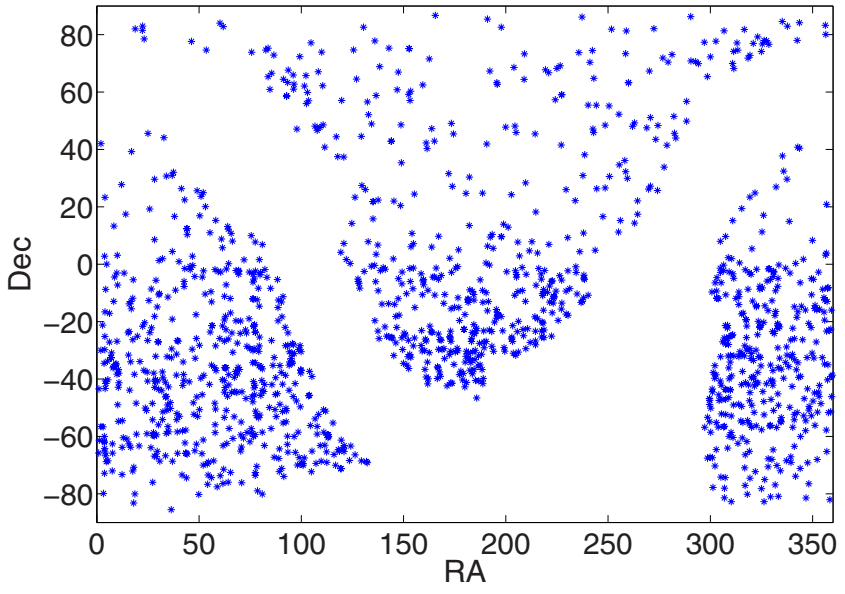

Figure 3. The RA and Dec of the final selection of M dwarf candidates.

NIR cuts can also be seen in Fig. 1. These regions isolate the general area of the M dwarf population however PPMXL also provides the photometry from USNO-B1 optical bands for many of its targets. Known M dwarfs were again used as a guide for the optical cuts and, when the colours were available, $\mathrm{K}$ dwarfs from the PMSU catalog to determine where $\mathrm{K}$ dwarfs lie in the colour space. All of the available colours were plotted against their spectral type. For the colours where the K dwarf information wasn't available, the cut was made on the blue end of the main body of the guiding samples to ensure completeness. The specific cuts can be seen in the upcoming paper. Objects with uncertainties in JHK photometry greater than 0.05 magnitudes were removed to limit saturated objects within the sample. Also objects with high proper motion uncertainties were removed using the relationship $\mu>5 \sigma$ where $\mu$ is the total proper motion of the object and $\sigma$ is the listed uncertainty. This obviously tended to remove objects that had a very low proper motion $(\mu<15$ mas $)$. Overcrowding was avoided by removing the galactic plane and other regions that had a high density of stars and dust. This may be added in at a later time using the appropriate reddening corrections and after accounting for crowding. 


\section{EPJ Web of Conferences}

\subsection{Reduced proper motion selection}

It has been shown previously by Lepine et al [1] that on occasions when distance to an object is not known, the reduced proper motion, $\mathrm{H}$, can be used as a proxy for absolute magnitude. $\mathrm{H}$ is defined as

$$
H_{k}=k+5 \log \mu+5,
$$

where $\mathrm{k}$ is the observed $\mathrm{k}$ magnitude and $\mu$ is in arcseconds/year. This can also be used to separate different kinematic populations between the disk and halo but the primary use within this work is to use it as a method to separate the dwarfs from the giants within our sample. Since the reduced proper motion is analogous to absolute magnitude, plotting $\mathrm{H}$ along with a colour index provides us with the equivalent of an HR diagram where the brighter giant branch is separated vertically from the dim red dwarfs. We use this, along with the giant and $\mathrm{M}$ dwarf guide samples defined above, as a way of identifying the different populations within our sample. We were able to take advantage of PPMXL's wide range of colour space to precisely define our reduced proper motion cuts.

\section{FINAL SAMPLE}

Comparing our sample to that of Lepine's, we are clearly able to probe down to a lower proper motion limit. In the final selection, there are $283 \mathrm{M}$ dwarf candidates below the 40 mas limit and, when cross matched with Lepine's sample, this selection method produced more than 1300 new bright M dwarf candidates.

\section{References}

[1] Lépine S., Gaidos E. 2011, AJ, 142, 138

[2] Roeser S., Demleitner M., Schilbach E., 2010, AJ, 139, 2440

[3] Skrutskie M. F., et al., 2006, AJ, 131, 1163

[4] Monet D. G., et al., 2003, AJ, 125, 984

[5] Ma C., et al., 1998, AJ, 116, 516

[6] Bochanski J. J., Hawley S. L., Reid I. N.,Covey K. R., West A. A., Tinney C. G., Gizis J. E., 2005, AJ, 130, 1871

[7] Reid I. N.,1997, yCat, 3198

[8] Jahreiß H., Gliese W., 1993, dgs..work, 53

[9] Riaz B., Gizis J. E., Harvin J., 2006, AJ, 132, 866

[10] Famaey B., 2005, A\&A, 430, 165

[11] Perryman M. A. C., et al., 1997, A\&A, 323, L49

[12] Sharma S., 2011, ApJ, 728, 106 